Kairos. Journal of Philosophy \& Science 20, 2018

Center for the Philosophy of Sciences of Lisbon University

\title{
More than Fitness. A Robustness-based Proposal of a Logical Space to Classify Processes Behind Evolutionary Phenomena
}

\author{
Giorgio Airoldi \\ Universidad Nacional de Educación \\ a Distancia (UNED), Spain \\ gairoldi1@icloud.com
}

\begin{abstract}
The assumption that natural selection alone is sufficient to explain not only which traits get fixed in a population/species, but also how they develop, has been questioned since Darwin's times, and increasingly in the last decades. Alternative theories, linked to genetic and phenotypic processes, or to the theory of complex systems, have been proposed to explain the rise of the phenotypic variety upon which natural selection acts. In this article, we illustrate the current state of the issue and we propose a logical space based on phenotypic robustness that allows a classification of evolutionary phenomena and can provide a framework for unifying all these accounts.
\end{abstract}

Keywords Philosophy of Biology, Evolution, Natural Selection, Organismic Design, Robustness.

DOI 10.2478/kjps-2018-0005

\section{Introduction}

The complexity of living beings has always stimulated the question about its origin. Against the traditional answers based on theological considerations, Darwin's theory is revolutionary for its approach more than for its content: the apparent intelligent design of organisms is not a problem to be solved, but a mirage to be dissolved. The theory of nat- 
ural selection expels God from the biological world in the same way as Laplace, hundred years earlier, had expelled it form the physical world: as a 'superfluous hypothesis'. Not from an intelligent act of creation, but "from the war of nature, from famine and death" (as we read in the Origin) spring the variety and the complexity of organisms.

While there is wide consensus about how natural selection shapes variety in populations (a process captured by population genetics formalism), the problems of explaining how traits appear in the first place and how organismic architecture changes are still open (Birch 2014). Adaptationist accounts defend that natural selection, through lineal, continuous and incremental changes led by a process of optimization of fitness, is the only relevant mechanism in place. Under this view, an organism is just the sum of its phenotypic traits, each contributing to its global fitness and selected thanks to such a contribution. Many critiques have been advanced to this paradigm, due, for example, to the lack of formal models demonstrating that fitness is optimized under natural selection, or to the unlikeliness that single mutations can have fitness-increasing effects. Several alternative processes have been proposed to explain how existing traits' morphology and function change, and how new traits appear. Such proposals get over the idea of organismic architecture as simple sum of traits.

We believe that the abundance of such explications is due to the existence of a wide range of evolutionary phenomena: different processes may act in some of them and not in others. To analyse which ones are relevant in each case, it is first necessary to classify these phenomena according to some criteria. To do so, we propose to add robustness as a second dimension to the adaptationist measure of organismic design based on fitness. We consider that many of the processes proposed as alternative to natural selection have effect mainly on robustness instead than on fitness. We can thus create a conceptual bi-dimensional space where it is possible to map the individuals of a population and to track its inter-generational movements due to any evolutionary force.

Section 2 presents the adaptationist account and the mains critiques to it found in the literature. Section 3 presents the main non-adaptationist proposals. Section 4 illustrates the proposed logical space and section 5 applies it to some examples. 


\section{Adaptationism}

Among the many ideas proposed by Darwin in the Origin, two are especially relevant regarding the relationship between natural selection and the phenotype. On one side, natural selection is the main cause of the differences among individuals of the same or of close species: such differences arise because they grant better chances of surviving and can be transmitted from generation to generation "Better chance of surviving thanks to inheritance of profitable variations", Ch. 3 of the Origin, 1872 ed.). On the other side, Darwin also thinks that natural selection leads to an increase in complexity of the organisms along the tree of life, thanks to the accumulation of these profitable variations ("Natural Selection acts exclusively by the preservation and accumulation of [beneficial] variations [...]. This improvement inevitably leads to the gradual advancement of the organization"; Ch. 4, section 9 of the Origin, 1872 ed.).

The relationship between natural selection (and other processes like migrations, mutations or drift) and the variety in a population are universally accepted and formalized by population genetics models. These models, however, focus on the genotype and do not especially commit with issues of phenotypic morphology. Moreover, they do not defend that fitness is maximized by natural selection: due to the complex relationship between genes, for example, a population's average fitness can decrease from one generation to the following (e.g. in case of overdominance of heterozygote over homozygote'). Population genetics is thus at best indifferent to Darwin's second claim, which is at the centre of the adaptationist research.

Adaptationism is a school of thought that justifies phenotypic traits through narratives based on the probable evolutionary history of the species, illustrating how each trait was selected thanks to its contribution to the fit between the individual and the environment. The adaptationist paradigm is so widespread among biologist (Orzack y Sober 2001, Sober 1998) that even Kimura, the founder of the neutral theory, while

1 In a population of $100 \%$ heterozygotes, the following generation will include some homozygotes. If the heterozygote is the fittest type, average fitness will decrease. 
claiming that natural selection has little impact at molecular level, affirms that "progressive evolution is almost always brought about as a result of organisms' response to environmental challenge", and that "evolution at the level of form and function is largely determined by Darwinian natural selection that brings about adaptation of organisms to their environment" (Kimura 1983:61-62, our italics). In its extreme version, adaptationism considers that all phenotypic traits are due to natural selection, a process external to the organism (seen as a passive object), lineal (as it affects each trait separately), continuous and progressive (as it proceeds by very small and swift changes between generations). Dennet underlines this view of natural selection as creative force acting on random changes when he writes: "Darwin's central claim is that when the force of natural selection is imposed on this random meandering, in addition to drifting there is lifting" (Dennet 1995). Under the adaptationist paradigm, phenotypic design is the sum of the traits of an organism, each contributing to the total fitness of the individual. Grafen gives an explicit definition in this sense: "Adaptation is design, and maximizing fitness is what organisms are designed for." (Grafen 2007). The slow and continuous action of natural selection sets up the best mix of such traits through a process of optimization of fitness. This idea is also referred to as the Positive View: natural selection explains not only the distribution of traits within a population, but also how they appear in the first place and how they set up phenotypic architectures (Neander 1995). Some formal models have been produced to support such claim, manly based on optimization programs (see, for example, Charnov 1976 for an application to foraging times; or Parker \& Maynard-Smith 1990 for an exhaustive list of models in the literature). The most ambitious attempt in this sense, although very controverted, is Grafen's Formal Darwinism Project (Grafen 1999, 2000, 2002, 2007, 2014a, 2014b). By applying population genetic formalism (in the form of the Price equation) to optimization programs, Grafen aims at demonstrating that fitness maximization under natural selection is a general trend. ${ }^{2}$

$\overline{2}$ Even if it is not realized due to genetic constraints such as overdominance. 
The most known critique to adaptationism is against its supposed 'panglossianism'3 (Gould y Lewontin 1979): it is always possible to build ex-post a narrative explaining why a species shows a trait, but these narratives are usually impossible to falsify. Mayr's statement that "one can never assert with confidence that a given structure does not have selective significance" (1963:190) is a paradigmatic example of such an attitude. The critique is based on Popper's denial that existentialist statements of the form $\exists x Q x$ (like 'there are unicorns') are scientific (Popper 2002: 47-50), because impossible to falsify: one can always go on searching indefinitely for a unicorn, as one can always search indefinitely for an adaptive explanation for any trait. Other critiques are directed against the main adaptationist hypothesis:

* Natural selection does not seem to be the only source of phenotypic novelties. The fact that species' classification is often based on non-adaptive traits suggests that other processes of speciation are at work (Haldane 1932: 113-114). Additionally, there are many examples of phenotypic traits acquiring new functions without being adaptations (that is, without their function being explained by their evolutionary history): Gould and Vrba define them as 'exaptation' (Gould \& Vrba 1982).

* The configuration of phenotypic traits is not necessarily linked to a process of fitness optimization, which, as already mentioned, is questioned by the formal results of population genetics models. Some authors interpret Darwin's idea not as the survival of the fittest, but of any individual fit enough (Maturana and Varela 1980), or as 'the non-survival of the not-enough fit' (Pigliucci 2008).

Some authors go one step further and doubt that natural selection can be the source of any real innovation at all: like artificial selection, it can shape existing traits and maintain them in a population, but cannot create new ones. The denial of any creative power of natural selection, known as Negative View, has a long tradition (a thorough list of authors

3 Professor Pangloss, in Voltaire's Candide, following Leibnitz's idea that we live in the best of all possible worlds, finds a justification for any misfortune. Gould y Lewontin apply the definition to adaptationist narratives because these, as Pangloss's reasoning, justify a-priori any existing trait as the best possible adaptation to the environment. 
that challenge the creative view of natural selection is presented in Razeto-Barry and Frick 2011). Hugo de Vries, one of the fathers of the modern synthesis, says that natural selection explains the survival, not the arrival of the fittest. Wagner (2015) believes that the real mystery of evolution is not the selection, but the creation of phenotypes. According to Moczek (2008), the last century has seen great advancements in the understanding of how phenotypic traits diversify, not in how they appear. Neader (1995) lists several arguments in favour of the Negative View. Here we mention just the 'counterfactuals' one: any organism exists because of the line of its ancestors, regardless of which other organisms existed besides, and of their destiny: a world with unlimited resources (thus with no selection) would be populated by all kind of creatures not eliminated by their less-than-optimal adaptedness.

Following the increasing doubts about the prominent role of natural selection in creating phenotypic novelties, many alternative, non-adaptationist ${ }^{4}$ processes have been proposed in the last decades. These proposals do not deny the role of natural selection, but aims at overcoming what they consider its limited potential for producing new traits. In the next section, we briefly illustrate some of the most debated.

\section{Alternative Proposals}

Instead of considering traits as the outcome of an external and progressive process led by the optimization of fitness, researches in molecular and evolutionary biology and complex systems behaviour suggest that the organism can produce novelties fuelled by internal forces, and that the timing of their rising can be substantially shorter than the timing of natural selection (see e.g. the punctuate equilibria hypothesis in Eldredge \& Gould 1972). We briefly introduce some among the many proposals found in the literature, underlying in each case at what level the process acts: in the genotype, in the phenotype, or in the whole organism, considered as a complex system.

$4 \quad$ In the sense that they do not rely on hypothesis of fitness optimization to explain phenotypic changes, not that they deny the importance of natural selection. 
Classical neo-Darwinian accounts consider mutations as the main source of novelties. Other accounts focus likewise in the genotype, but do not rely on the mechanism of single mutations. The Shifting Balance theory by Wright (1982) introduces the concept of genetic drift: genic frequencies between generations change not only under the effect of natural selection, but also for aleatory causes (e.g. accidental death of theoretically fitter phenotypes). Some alleles can thus get lost, while others get fixed, without any causal link to their contribution to individual fitness. This especially happens in small populations and, together with the complex relationship between genotype and phenotype, can lead to sudden modification of phenotypes. These aleatory modifications usually result in a decrease of average fitness, but can allow the population to explore parts of the adaptive landscape otherwise inaccessible, for being on the other side of a fitness valley. ${ }^{5}$

Another process allowing the appearance of phenotypic novelties in a non-progressive and non-lineal way is based on neutral genetic networks (Wagner 2007, 2011; Moczek 2008). Given a set of genes, there are different config.rations of them that produce the same phenotypic function. This means that, even if one of the genes suffers a mutation, the resulting phenotype remains unaffected. All conFig.rations maintaining the same function constitute a neutral genetic network. Thanks to this property of functional invariance, such networks allow the exploration of the logical genotypic space and the accumulation of cryptic mutations that, even if individually neutral, could together result in advantageous phenotypic novelties. Such novelties appear thus thanks to a process that does not have to do with natural selection, nor with fitness optimization, and whose effects are not progressive, nor lineal.

$5 \quad$ Wright (1982) creates the metaphor of adaptive landscape to show how natural selection tends to increase the average fitness of a population but do not assure that fitness reaches its theoretical maximum. Interpreting the Cartesian function linking genetic configuration and fitness as a landscape full of peaks and valleys, Wright considers that populations tend to climb toward the closest peak (because of Fisher fundamental theorem, Fisher 1930). Once reached the fitness peak, the population cannot descend if natural selection is the only process acting on it. Given that, in general, there are local peaks that do not coincide with the highest possible fitness, a population on a local peak cannot reach a higher peak crossing a valley. 
Functional novelties can also appear thanks to existing traits, without any genetic change (at least initially): exaptations are the best-known example (Gould \& Vrba 1982). An exaptation is an existing trait that, regardless of how it appeared (i.e. regardless of it having a previous adaptive function), ends up contributing positively to the individual fitness due, for example, to some change in the environment. Feathers, initially a thermoregulation tool, and bones, initially a calcium-storage element, are examples of exaptations.

Finally, the source of phenotypic novelties can be the whole organisms as a system (genotype, phenotype and all interrelationships between the two). Against the adaptationist view of the organism as a passive entity shaped by the environment, the theory of complex systems considers that its structure also depends on development laws and self-organization principles. According to Kauffman (2000), all complex systems are governed by the same universal laws that lead to increasing variability and complexity. McShea $\&$ Brandon (2010) defend the same idea when they underline the tendency of organisms to become more and more diversified and complex ${ }^{6}$ even in the absence of any external evolutionary force: tendency that they identify as the 'zero-force-evolutionary-law'. A complex system positions itself in the logical space of state variables around stable points called 'attractors'. If, following an external perturbation, the stable system moves away from the attractor, it will return to it or to move to another one, without dissolving in the process (Kitano 2004, 2007). If we interpret an attractor as a viable phenotype, its viability rests on internal principles and not on natural selection. Modifications in a phenotype can appear by sudden 'jumps' to a new attractor, and not gradually.

Evo_Devo ${ }^{7}$ research investigates the 'developmental laws' leading the embryo to adult state, and that seem to remain constant throughout

$6 \quad$ It is worth noting, though, that the concept of complexity in McShea and Brandon is quite different than in Kaufmann, and considers just the number of parts of an organism, regardless of their functions.

$7 \quad$ Evo-Devo is the abbreviation for Evolutionary Developmental Biology. By comparing the developmental process of different organisms to determine their phylogenetic relationships, Evo-Devo research tries to identify the mechanisms that cause evolutionary changes in phenotypes (Hall, 2003a). 
phylogenetic evolution. Possible morphologies depend not only on the genotype, but also on these laws (Alberch 1991, Callebaut \& Rasskin-Gutmann 2005, Gould \& Lewontin 1979). Development can heavily depend on environmental conditions (e.g. sex determination in some reptiles is linked to external temperature), thus the same genotype can result in very different phenotypes. The Evo-Devo proposal is in some way complementary to the complex system approach: while the latter focuses on the equilibrium points of complex systems, the former determines the potential path between attractors: not all morphologies are viable, and not all paths between morphologies are possible.

In the present section, we have briefly discussed just some of the many processes proposed as alternative to natural selection to explain how new traits appear in organisms. We consider that the abundance of such proposals is due to the existence of a great variety of different evolutionary phenomena. In the following section, we suggest a classification of such phenomena that allows to identify which process acts, and how, in each case.

\section{Elements of Biological Design}

\subsection{Variety of Evolutionary Changes}

Evolutionary phenomena encompass a great variety of cases, from micro-evolution to speciation, from the polymorphism in populations to the appearance of new traits and functions. We analyse three cases that are intermediate in this range of possibilities, specifying what the change consist of in terms of traits and functions, and what the role of natural selection might have been in shaping it (examples are partially inspired by Pigliucci 2008).

Changes in the wings' colour of $B$. betularia following the increasing pollution for coal smoke in the proto-industrial England is an example of evolution led by natural selection. Originally, individuals had mainly white wings (morpha typica) to camouflage efficiently on the white bark of poplar. Due to the increasing pollution, barks turned dark, and individuals with white wings became easy target for predators, while the mutation correspondent to black wings (morpha carbonaria) spread quickly through the population. The trait itself (the wing's colour) and 
its function (mimicry) did not change. The black version of the trait already existed, either actually, although with a very low frequency; or as a potential recurrent mutation (as it seems to be the case, see Van't Hoff et al. 2016). The evolutionary phenomenon consists thus just in the change of the relative frequencies of the trait versions, led by natural selection: the black wing version became more frequent because it granted a higher fitness.

Finches' speciation in the Galapagos islands seems a different case. The opportunity to access new nutritious resources in each island gave raise to new versions of the beak's morphology, allowing to perform new functions (e.g. reaching insects in places inaccessible with a short beak). Although it is undeniable that natural selection played an important role in this process, the appearance of the variants might be due to some of the other processes presented in the previous section.

Finally, new traits and functions, like flight, or the transformation of an existing trait in a new one, like the turtle carapace, often involve the need for a new phenotypic architecture: universally accepted accounts of such phenomena based uniquely on fitness changes and natural selection are not available. Other processes might play a role in creating such novelties, even though natural selection determines, once appeared, their fixation in the population.

\subsection{Dimensions of Design}

Based on these simple examples, we believe that, to understand which processes lies behind an evolutionary phenomenon, it is necessary to build a classification to distinguish the effects of natural selection upon the population average fitness from the effects of other evolutionary processes that do not impact primarily and directly upon fitness.

As we have seen in section 2, adaptationism, both in its narrative accounts and in its formalizations, reduces any phenotypic change to a fitness change. Without taking into account architectural considerations, it equals design with mix of traits, in the same way as it calculates individual fitness as the sum of the fitness granted by each single trait. An improvement in design is measured as increase in fitness: but fitness (at least as biologist usually define it) summarizes the success of a design by measuring its results, without investigating the reasons 
behind them. Under this approach, the organism itself remains a blackbox (Hall 2003b) whose internal mechanisms are unknown and, at least in the adaptationist formal models, of little interest. Such a vision, adequate in cases like the $B$. betularia wings' colour changes, is too simple to be able to capture the complexity of evolutionary phenomena involving new traits and architectures. To understand these, it is necessary to 'open' the black-box.

The limitation of fitness as only explanans for phenotypic traits is evident in the circularity that makes it impossible to define independently the concepts of fitness, survival and reproduction (Mills \& Beatty 1979). We believe that this circularity is due to the polysemy of the term fitness, interpreted at the same time as cause and effect of differential reproduction (Rosenberg \& Williams 1986): polysemy that lies behind the known tautology problem of the theory of natural selection ${ }^{8}$. Many solutions have been proposed to refine the definition of fitness and escape the tautology. Sober (2009) divides fitness between the ability to survive in an environment (or ability to pass from zygote to adult, that he calls 'viability'), and the ability to reproduce (or ability to pass from fertile adult to zygote, that he calls 'reproductive fitness'). Although a very clarifying distinction, both concepts measure evolutionary facts ex-post. Using a metaphor, it is like saying that the best designed car is the one that won the race (viability), or that the one with the most copied technology (reproductive fitness): while probably true, these sentences cannot help explaining why the car won, or why the technology is so widely copied. The propensity interpretation of fitness (Mills \& Beatty 1979) shifts the focus from the actual to the expected number of offspring of an individual, whose value is not defined within the natural selection theory but depends on 'casually relevant features' of the organism, to be identified based on considerations from other disciplines (e.g. engineering considerations). This dependence of the definition of fitness from external theories has been used to claim that fitness is a 'primitive theoretical term', undefinable within the theory of natural selection (Rosenberg 1982 , Rosenberg \& Williams 1986). Millstein (2006) proposes likewise to

8 If the fittest survives (effect) and the fittest is the one who survives (cause), the theory just affirms that the one who survives, survives. 
substitute the term fitness with 'causally relevant physical differences', whose relevancy can only be defined through other disciplines. Although these proposals are ex-ante, they still focus on reproductive success, thus on the 'selection step' of evolution.

We therefore propose to integrate the unidimensional measure of organismic design as reproductive fitness with a second dimension that reflects ex-ante the capacity of the organism to face and survive new environmental challenges, and that is independent from the organism subsequent reproductive success: this dimension is phenotypic robustness.

\subsection{Phenotypic Robustness}

There are many definitions of robustness in the literature. Waddington (1957) was probably the first one to investigate what he calls 'canalization', by observing that organisms undergoing environmental shock show higher phenotypic variation. More recently, Moczek (2008) claims that novelties appear thanks to the canalization of developmental processes, i.e., the ability to maintain the phenotype against changes in genetic/environmental input: the accumulation of genetic variation over a threshold leads to new phenotypes. Kitano (2004) defines robustness of a system as its ability to return to the same attractor or to move to a new one without dissolving as a response to a perturbation. Carlson and Doyle (2002) identify robustness as a key element in complex systems and organizations, and define it as the ability to handle forecasted perturbations. Pigliucci (2008) defines the propensity to develop new traits and functions as 'evolvability' in a strict sense. Evolvability includes characteristics such as modularity, robustness and the genotype-phenotype mappings.

There are two aspects of robustness in these definitions, both captured by Wagner (2015):

a) the ability to resist changes in the current environment, thus keeping the organism alive.

b) the disposition to develop new traits, functions or architectures to adapt to new environments (e.g. a new niche), thus allowing changes in genotype/phenotype without losing the original functions. 
These two aspects refer to different approaches to the concept (Daniels et al. 2008):

a) Phenotypic robustness is the ability to resist change. It maintains functions against environmental perturbations. It can be measured as the average effect of a perturbation on the phenotype (Kitano 2007). This is what we consider as second dimension of design.

b) Genetic robustness is the disposition to develop new traits and functions. It maintains current functions against genetic perturbations (e.g. mutations) while allowing exploration of new functions/morphologies. It is thus part of the mechanism of canalization, as it allows the accumulation of cryptic genetic changes. It can be measured as the probability of neutral mutations (Draghi \& al. 2010).

We can also distinguish between robustness as:

* Property of a single trait performing some function, e.g. an enzyme robust against changes in temperature. It can be measured as the range of external variable in which the trait function is maintained. Proofreading is an example of a process increasing robustness in this sense. Its fitness equivalent is the ecological fitness of a trait.

* Dynamic feedback among interacting elements of a system, e.g. alternative metabolic routes. It can be measured as the probability of survival to an external perturbation. Redundancy is an example of system robustness. Its fitness equivalent is the individual or reproductive fitness.

The robustness of a system depends on some architectural elements, among which (Kitano 2004):

* System control: mechanisms of positive and negative feed-back (e.g. bacterial chemiotaxis, that governs the movement of bacteria due to chemical stimulus)

* Redundancy: different components that can replace each other (e.g. double organs)

* Diversity: several means to perform the same function (e.g. glycolysis and oxidative phosphorylation to produce ATP)

* Modularity: physical, functional and temporal modules that limit perturbations and damage locally (e.g. cells) 
* Decoupling: isolation of low-level variations from high level functionalities (e.g. genetic buffering).

Masel and Siegal (2009) add also cooperativity between parts, regulatory buffering (e.g. effect of temperature on transcription linked to competing activation/repression factors), and existence of switch-like responses ('transistor effect').

Once defined the two dimensions of design (reproductive fitness and phenotypic robustness), it is possible to map the individuals of a population into a logical space, and to track their inter-generational movements in response to different evolutionary processes, once some hypothesis are made around the impact of such processes upon the two dimensions.

\section{Design Space}

\subsection{Elements}

Fig. 1 shows the proposed logical space.

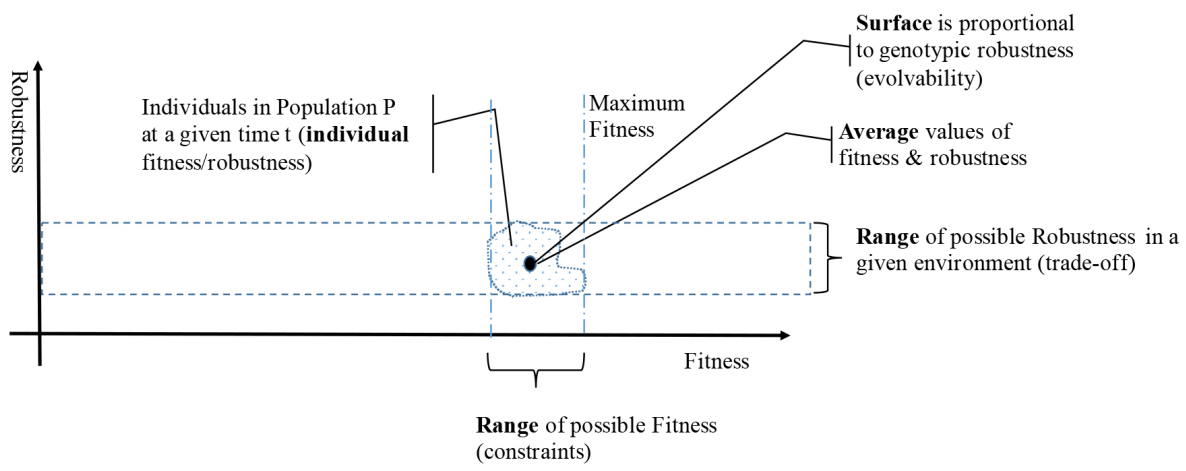

Fig. 1 - Design logical space

The punctuated area represents a population, whose individuals have different fitness and robustness. Maximum theoretical fitness is determined by genetic, historical and developmental constraints, while upper and lower limit of robustness are linked to the trade-off between the 
advantages of a higher robustness and its costs (Wagner 2015, Lenski et al. 2006, Kitano 2004). Average fitness and robustness are placed somewhere within the area, depending on how they are defined. Finally, the surface of the area is somehow linked to the genetic robustness of the population: the highest it is, the farther the population can spread around the average phenotype. The area included between maximum and minimum of fitness and robustness is the equivalent of an attractor in the sense of complex system theories: individual within this area are stable, thus viable.

In this logical space, any evolutionary phenomenon is represented by a movement of the area or a change in its shape. Natural selection and the alternative processes have peculiar impacts on the average and the variance of one or both dimensions: fitness increases if reproductive success augments; robustness increases if any modification in the architecture or in the functions of the phenotype improves survival.

We postulate that changes in fitness and changes in robustness are quite independent, given that evolution by natural selection is a two-step process: creation of traits followed by selection of traits. This assumption is neutral with regards to the positive/negative view debate: Neader (1995), for example, in favour of the former, thinks that the cumulative process of creation/selection is itself a fundamental piece of the generation of novelties, but does not deny that the two steps exists: novelties are built by a series of vertical and horizontal movements (increase in robustness and in fitness), although each very small.

We also postulate that changes in fitness are mainly linked to selection, while changes in robustness are mainly due to non-selective processes. For example, a new trait (e.g. a duplicated organ) appears as consequence of cryptic genetic changes, thus affecting the phenotypic robustness; but whether the trait will get fixed or not will be decided by natural selection. We propose to see improvement in fitness as ways to do the same, with the same resources, but better and more efficiently; and improvement in robustness as ways to do the same with new resources, or to do something new, but not necessarily better: natural selection will decide whether the new resources or the new functions bring any competitive advantage or not. 


\subsection{Effects of Evolutionary Forces}

Fig. 2 (a-e) show, in the proposed logical space and under our hypothesis, how natural selection and other non-selective processes, when acting alone, affect fitness and robustness of a population (both in terms of average and of variance).

Following Fisher fundamental theorem (Fisher 1930, Price 1972), average fitness will never decrease as consequence of the action of natural selection alone ${ }^{9}$, while its variance decreases. These are the cases in which a new trait, however appeared, spreads and gets fixed in a population (e.g. the $B$. betularia wings' colour), or the value of a quantitative trait gets optimized. On the other hand, we postulate that average robustness does not change significantly under the effect of natural selection. The reason is that phenotypic functions do not change, so according to Kitano (2004) robustness does not change either. Under this hypothesis of constant robustness, formal models based only on fitness changes and considering only natural selection work well (e.g. Grafen's project or optimization models) (Fig. 2a).

The 'shifting balance' effect is a continuous, but non-directional process (as it depends on drift, which is not directional, see e.g. Rice 2004). It affects both average fitness and robustness, increasing or decreasing them through casual sampling, while not affecting their variances (McShea and Brandon 2010) (Fig. 2b).

The 'Zero Force Evolutionary Law' effect, on the contrary, increases variances, but leaves averages unaffected. It is therefore non-directional, but in a different way than drift: while drift causes averages to wander without forecastable direction, the ZFEL effect increases the spread around constant averages (McShea and Brandon 2010) (Fig. 2c).

Exaptations can be interpreted as increasing robustness by definition because, if not, they would not be exaptations. This increase is discrete, in the sense that an already available phenotypic trait 'suddenly' becomes useful to cope with, for example, a new environmental challenge. Their impact on fitness is null: only the subsequent process of natural selection

$9 \quad$ Average fitness might decrease if processes other than natural selection also act, e.g. environmental changes, mutations, etc. 


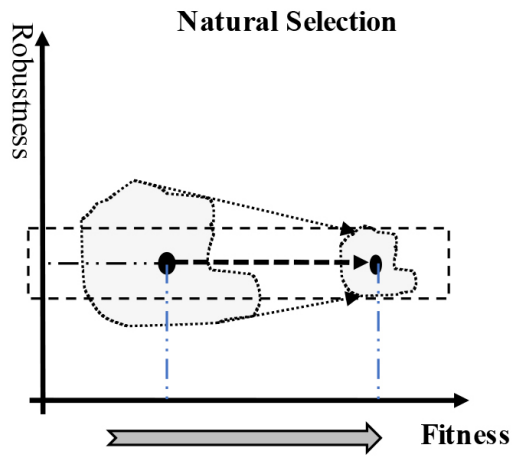

Fig. 2.a - Natural Selection pushes the population toward higher average fitness and reduces fitness variance, while having limited impact on robustness.

Fig. 2.c - The ' Zero Force Evolutionary Law'
leaves averages unchanged, while increasing
fitness and robustness variance.

Fig. 2.c - The ' Zero Force Evolutionary Law'
leaves averages unchanged, while increasing
fitness and robustness variance.

Fig. 2.c - The ' Zero Force Evolutionary Law'
leaves averages unchanged, while increasing
fitness and robustness variance.

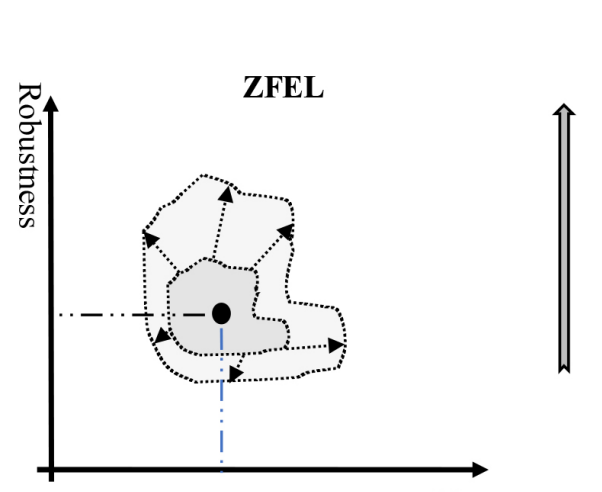

Fitness

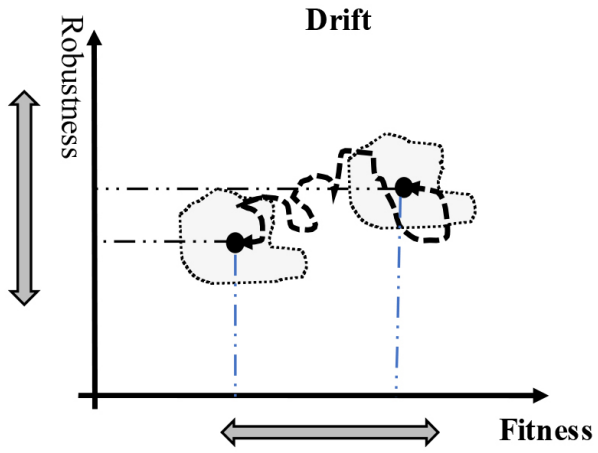

Fig. 2.b - Drift causes fitness and robustness random changes, both in average and in variance.

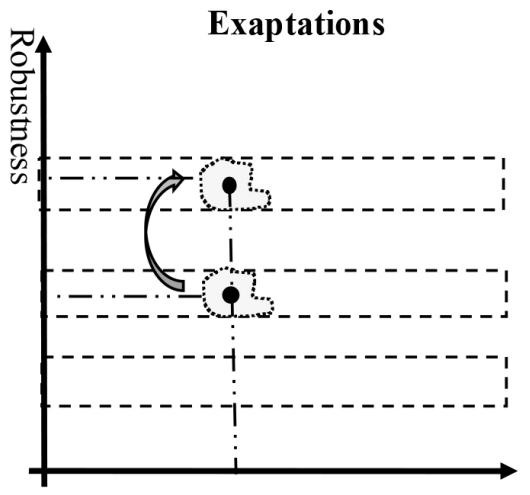

Fitness

Fig. 2.d - Exaptations have no immediate effect on fitness, while increasing robustness.

will decide whether the novelty is efficient enough to get fixed throughout the population, or if it is a resource-consuming robustness increase that does not impact fitness and might disappear (Fig. 2d).

Complex systems' self-organizing rules affect both the average and the variance of robustness, given that they tend to increase the complexity of the system in the sense of new parts, new connections between parts and new functions (Fig. 2e). 


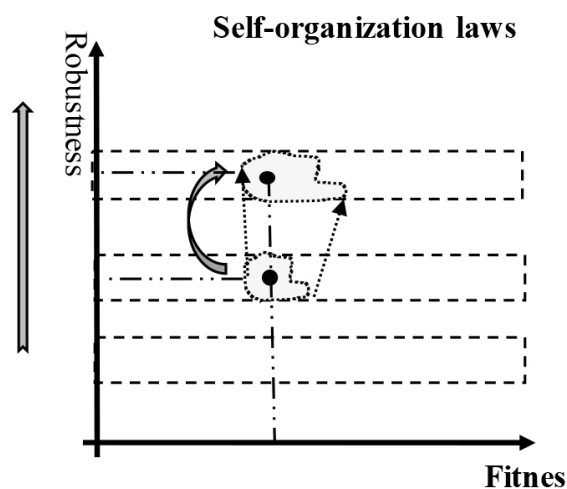

Fig 2.e - Complex systems laws affect both average and variance of robustness.

In the following paragraph, we apply this theoretical framework to two evolutionary phenomena of different nature.

\subsection{Examples}

First, we analyse what we consider a pure selection process: the change in wings' colour of the $B$. betularia. It is an evolutionary phenomenon that adaptationism can easily explain: there are neither modifications in the organismic architecture nor new traits. Following an environmental change, the fitness of some phenotypes (the carbonaria black wing individuals either already existent in low frequencies or appearing following a recurrent mutation) increases, while the fitness of the white coloured individuals decreases. The population, after an initial contraction of volume, returns to the same state it had before the change, but with a majority of black wings individuals. Given that no change in robustness occurred (at least, in the sense of robustness we apply), natural selection alone can explain the phenomenon, and fitness summarizes changes in design. For this reason, formal accounts like Grafen's Formal Darwinism Project successfully apply. Fig. 3 shows the steps of the phenomenon.

In the case of the appearance of the function of flight, the evolutionary phenomenon is more complex. Although natural selection doubtless played an important role, other processes contributed to the creation of fundamental architectural elements like feathers (see for example Prum 1999). We propose the following narrative just to show a possible 


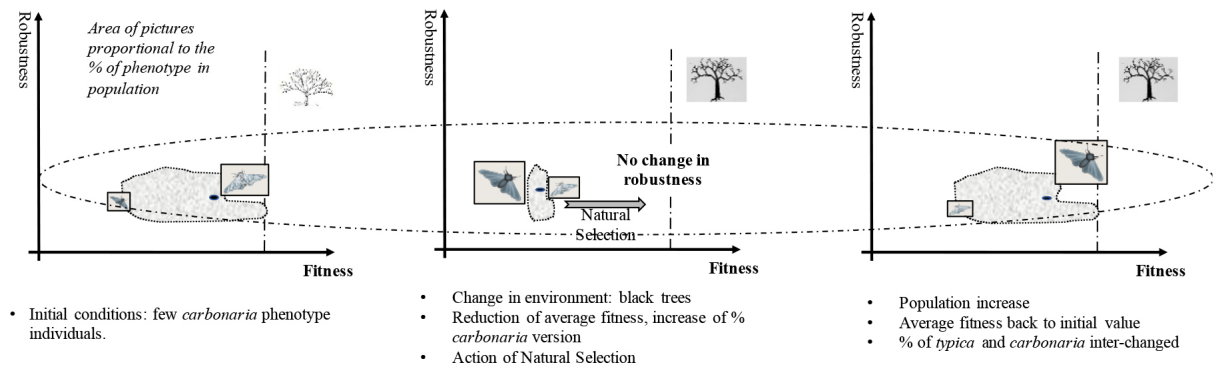

Fig. 3 - Adaptation as answer to an environmental change, without change in robustness. (Areas of rectangles is proportional to the number of individuals).

series of events behind the evolution of the new function, without any ambition that they reflect the actual ones. The first step consisted in the appearance of the new trait 'feathers' caused, for example, by drift and accumulation of cryptic mutation. This new trait allowed for better thermoregulation, thus increasing robustness (vertical upward movement), as the modified phenotype was able to resist a wider range of external temperatures (Fig. 4a). The higher robustness could or could not grant an adaptive advantage, and its impact on fitness was decided by natural selection, that eventually spread it among the individuals of the population and optimized the trait (e.g. shape and quantity of the feathers horizontal movement) (Fig. 4b). At some point, the new trait became an exaptation, allowing a primitive and rudimentary flight. Robustness increased again, but not necessarily fitness (vertical upwards jump): if flight had not granted any advantage, natural selection could have eliminated it (Fig. 4c). As it did grant an advantage, natural selection fixed the corresponding traits in the population (horizontal movement) (Fig. 4d). The global movement from the initial to the final phenotype was therefore the sum of several horizontal and vertical movements, each fuelled by a different evolutionary process, not always and not only measured by increases in fitness. 


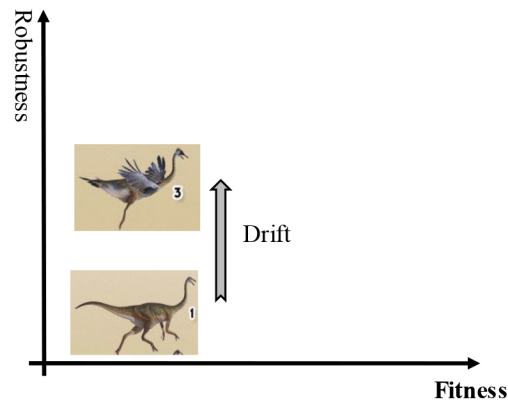

Fig. $4 a-a$ new trait (feather) appears due e.g. to drift: robustness increases.

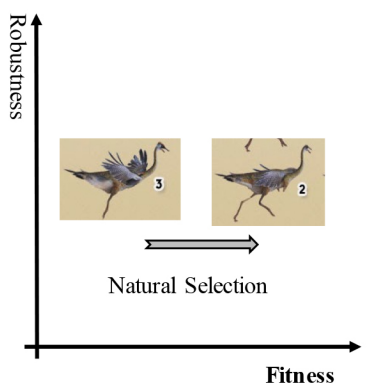

Fig. $4 b$ - the new trait gets fixed and is optimized by natural selection: average population fitness increases, while average robustness is unaffected.

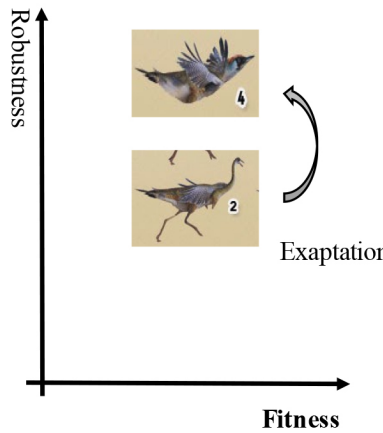

Fig 4c - At a certain point, the new trait becomes an exaptation and causes a jump in robustness.

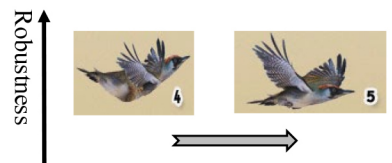

Natural Selection

Fig. $4 d$ - The 'exaptated' trait gets fixed and is optimized by natural selection: average fitness increases, with no further increase in robustness.

\section{Conclusions}

In the last decades, many processes alternative to natural selection have been proposed to explain how new phenotypic traits arise. This abundance is partially due to the great variety of different evolutionary phenomena. In this article, we propose an extension of the measurement of biologic design that adds phenotypic robustness to reproductive fitness. In the bi-dimensional logical space so defined, it is possible to map the individuals of a population and to track the effects on their fitness and robustness of different evolutionary processes, clarifying which one acts in which cases, and how. 


\section{References}

Alberch, P. (1991). From genes to phenotypes: dynamical systems and evolvability. Genetica 84, 5-11.

Birch, J. (2014), "Has Grafen formalized Darwin?", Biol Philos, 29: 175-180.

Callebaut, W., Rasskin-Gutman, eds. (2005). Modularity. Understanding the Development and Evolution of Natural Complex Systems. The MIT Press.

Carlson, J.M., Doyle, J. (2002). Complexity and Robustness. PNSA, vol. 99, 2538-2545.

Charnov, E.L. (1976). Optimal Foraging, the Marginal Value Theorem, Theoretical Population Biology, Vol. 9, No. 2.

Daniels, B.C. et al. (2008), Sloppiness, robustness and evolvability in systems biology, Current Opinion in Biotecnology, 19: 389-395.

Dennet, D.C. (1995). Darwin's Dangerous Idea, Penguin Books.

Draghi, J.A. et al. (2010). Mutational robustness can facilitate adaptation, Nature, 463: 353-355.

Eldredge, N. \& Gould, S.J. (1972), Punctuated equilibria: an alternative to phyletic gradualism, in Models in paleobiology, Shopf, TJM Freeman, Cooper / Co.

Fisher, R.A. (1930). Genetical theory of natural selection. S.I.: Oxford Clarendon Press.

Gould S.J., Lewontin S.J. (1979), The spandrels of San Marco and the Panglossian paradigm: a critique of the adaptationist program, Proc. $R$. Soc. Lon., B 205, 581-598.

Gould, J.S., Vrba, E.S. (1982), Exaptation - A Missing Term in the Science of Form, Paleobiology, Vol. 8,No. 1: 4-15.

Grafen, A. (1999) "Formal Darwinism, the individual-as-maximizing-agent and bet-edging", Proc. R. Soc. Lond. B., 266, 799-803.

--- (2000) "Development of the Price equation and natural selection under uncertainty" Proc. R. Soc. Lond. B., 267, 1223-1227.

--- (2002) "A first formal link between the Price equation and an optimization program", J. Theor. Biol. 217, 75-91. 
--- (2007) "The Formal Darwinism project: a mid-term report", J. Evol. Biol., 1243-1254.

--- (2014a) "The formal Darwinism project in outline", Biol Philos, 29: 155-174.

--- (2014b) "The formal Darwinism project in outline: response to commentaries”, Biol Philos, 29: 281-292

Haldane, J.B. (1932). The Causes of Evolution. Longmans Green.

Hall, B.K. (2003a). Evo-Devo: evolutionary developmental mechanisms. Int. J. Dev. Biol. 47: 491-495.

--- (2003b). Unlocking the Black Box between Genotype and Phenotype: Cells and Cell Condensations as Morphogenetic (modular) Units. Biol. \& Philos., 18: 219-247.

Kauffman, S., (2000). Investigations. Oxford University Press.

Kimura, M. (1983). The neutral theory of molecular evolution. Cambridge University Press.

Kitano, H. (2004). Biological robustness. Nature Reviews Genetics, 5(11), 826-837. doi:10.1038/nrg1471.

--- (2007). Towards a theory of biological robustness. Molecular Systems Biology, 3(1), 137-n/a. doi:10.1038/msb4100179.

Lenski, R.E., Barrick, J.E., \& Ofria, C. (2006). Balancing robustness and evolvability. PLoS Biology, 4(12), e428. doi:10.1371/journal. pbio.0040428

Masel, J., \& Siegal, M.L. (2009). Robustness: Mechanisms and consequences. Trends in Genetics, 25(9), 395-403. doi:10.1016/j. tig.2009.07.005.

Maturana, H., \& Varela, F.J. (1980). Autopoiesis and cognition: The realization of the living. Dordrecht: $\mathrm{D}$. Reidel.

Haldane, J.B. (1932). The Causes of Evolution. Longmans Green.

McShea, D., Brandon, R. (2010). Biology's First Law: the tendency for diversity and complexity to increase in evolutionary systems. The University of Chicago Press.

Mills, S., Beatty, J. (1979), The Propensity Interpretation of Fitness, Philosophy of Science, 46: 263-286. 
Millstein, R.L. (2006). Natural selection as a population-level causal process. British Journal for the Philosophy of Science, 57(4):627-653.

Mocczek, A.P. (2008). On the origins of novelty in development and evolution. BioEssays 30: 432-477.

Neander, K. (1995). Pruning the Tree of Life. The British Journal for the Philosophy of Science, 46(1): 59-80.

Orzack, S.H. \& Sober, E.R., eds. (2001). Adaptationism and Optimality. Cambridge University Press.

Parker, G., Maynard Smith, J. (1990). Optimality theory in evolutionary biology, Nature, 348: 27-33.

Pigliucci, M. (2008). Is evolvability evolvable? Nature Reviews Genetics, 9(1), 75-82. doi:10.1038/nrg2278.

Popper, K. (2002). The Logic of Scientific Discovery. Routledge.

Price, G.R. (1972). Fisher's 'fundamental theorem' made clear. Annals of Human Genetics, 36(2), 129.

Prum, R.O. (1999). Development and evolutionary origin of feathers. J. Exp. Zool. B Moi. Dev. Evol. 285: 391-306.

Rice SH (2004) Evolutionary Theory: Mathematical and Conceptual Foundations. Sinauer Associates, Sunderland, MA.

Rosenberg, A. (1982). On the Propensity Definition of Fitness. Philosophy of Science, Vol. 49, No. 2:268-273.

Rosenberg, A., Williams, M. (1986). Fitness as Primitive and Propensity. Philosophy of Science, Vol. 53, No. 3:412-418.

Sober, E. (1998) Six Sayings about Adaptationism in D. Hull and M. Ruse (eds) The Philosophy of Biology. Oxford University Press.

--- (2009). Philosophy of biology. New York: Westview Press.

Van't Hoff et al. (2016). The industrial melanism mutation in British peppered moths is a transposable element. Nature 534: 102-105.

Waddington, C.H. (1957). The Strategy of the Genes. George Allen \& Unwin.

Wagner, A., (2007). Robustness and Evolvability in Living Systems, Princeton University Press. 
More than Fitness.

A Robustness-based Proposal of a Logical

Space to Classify Processes Behind Evolutionary Phenomena

--- (2011), The Origins of Evolutionary Innovations, Oxford University Press.

--- (2015). Arrival of the Fittest, Oneworld Publications.

Wright, S. (1982), The shifting balance theory and macroevolution, Ann. Rev. Genet. 16:1-19. 